\title{
THE CONDITIONS OF CONJUGATION IN THE TASKS OF MOISTURE TRANSFER ON A THIN CLAY INCLUSION TAKING INTO ACCOUNT SALT SALUTIONS AND TEMPERATURE
}

\author{
YURiI Chui, PETRO MARTYNIUK*, \\ Mykola KuZlo, OKSANa UlianchuK-MARTYNiUK \\ National University of Water and Environmental Engineering, Rivne, Ukraine \\ [Received 21 September 2018. Accepted 18 February 2019] \\ doi: 10.7546/JTAM.49.19.01.03
}

\begin{abstract}
The processes of non-isothermal moisture transfer of singlecomponent chemical solutions in the porous environment containing thin semipermeable clay inclusions have been considered. The conjugation conditions for the functions of humidity, salt concentration and temperature have been modified. The modification has been based on taking into account the dependences of the parameters of the porous medium on the physico-chemical processes and osmotic flows.
\end{abstract}

KEY WORDS: moisture, salt solutions, temperature, porous medium, semipermeable membrane, conjugation condition, osmosis.

\section{INTRODUCTION}

When designing and constructing waste storage facilities in their soil bases antifiltering artificial inclusions are created. The task of these anti-filtering screens is to prevent or minimize the spread of pollution on the environment. Some types of clays are used as natural materials for such inclusions. Such clays possess properties of semi-permeable membranes and they have characteristic osmotic properties.

Under the conditions of the mathematical description of the moisture transfer in soil areas with thin semi-permeable inclusions, one of the important tasks is the derivation of conjugation conditions. The conjugation conditions $[1,2]$ are the part of the boundary value problem in non-homogeneous domains, by which the constructed mathematical model is described. Their presence is mandatory in terms of the correctness of such tasks.

In the works [3-5] such conditions for the problems of moisture transfer are designed. However, the characteristics of the inclusion material were considered constant and not dependent on the concentration of salt solutions and temperature. It is well known that the properties of liquid clay permeability depend considerably on

\footnotetext{
${ }^{*}$ Corresponding author e-mail: p.m.martyniuk@ nuwm.edu.ua
} 
these factors. Therefore, our goal is to deduce such conjugation conditions for the problem of moisture transfer when the characteristics of the medium depend on the characteristics of the physical and chemical processes in these environments.

At present in research literature, there is considerable progress on the study of semipermeable properties of clay inclusions, both for complete and incomplete saturation.

In the works $[6,7]$ a special kind of bentonite, which is used in waste geobarriers to limit the leakage of harmful substances to the environment has been considered. As shown earlier by the authors of the article - saturated bentonite can display the properties of semipermeable membranes, thereby increasing the productivity of the barrier. However, as shown in the above articles, the effect of the bentonite membrane on the transport of contaminated substances is more significant for unsaturated conditions. This fact confirms again the relevance of the modification of conjugation conditions precisely for the tasks of moist transfer.

In the work [8] the behavior of membranes from bentonite soil compositions used for walls of waste disposal facilities has been studied. Two semi-permeable models, namely, natural clay and a mixture of pure sand with $5 \%$ of the mass fraction of pure bentonite, which exhibit the properties of semipermeable membranes have been considered. It has been shown that the chemical-osmotic efficiency for natural clay varies from 0.018 to 0.024 , whereas for a sand-bentonite mixture, this value ranges from 0.118 to 0.166 . It has been established that the use of sand-bentonite mixture will reduce the transfer of contaminants by $10 \%$ in comparison with the case when materials with properties of semi-permeable membranes are not used at all.

In Ref. [9] geomembranes and geosynthetic clay layers used as part of complex waste management systems have been considered. The results of experiments of spatial and temporal distribution of moisture in these systems in isothermal conditions have been presented.

In Ref. [10] the phenomenon of ultrafiltration, which is characteristic for environments with semi-permeable and osmotic properties has been investigated. Onedimensional transfer of $\mathrm{NaCl}$ solution through clay membrane layers with the use of new data about geological properties of membranes has been considered. New effects of ultrafiltration have been investigated.

In the work [11] a new system of equations that describes the transfer of ions and water through a porous medium in the case of incomplete saturation has been presented. The case of reverse osmosis and the value of the diffusion coefficient have been analyzed in detail for the case of saturated and partially saturated porous materials.

The work [12] focuses on the diffusion of potassium chloride $(\mathrm{KCl})$ through a bentonite-polymer composite that acts as a semipermeable membrane. The negative 
influence of chemicals on the productivity of bentonite hydrobarriers has been investigated. It has been established that the diffusion coefficient for chlorine $\left(\mathrm{Cl}^{-}\right)$and potassium $\left(\mathrm{K}^{+}\right)$is correlated with the coefficients of membrane efficiency.

In the work [13] clay layers that can display the properties of semipermeable membranes have been considered.

In the work [14] the macroscopic description of osmotic phenomena in clay soils has been given. Theoretical descriptions of two potential mechanisms for changing the volume of porous medium - consolidation and osmotic filtration consolidation have been presented. Osmotic consolidation occurs due to changes in electrostatic stresses between clay particles. Osmotic filtration consolidation occurs due to osmotic outflow of fluid from clay, as a result of osmotic gradients. The results of laboratory studies on two clay soils under the influence of concentrated $\mathrm{NaCl}$ solutions have been used to illustrate that osmotic consolidation is the dominant mechanism of volume change in clay soils.

As mentioned before, when constructing mathematical models of geomorphic processes in environments with inclusions, the corresponding boundary value problems must be correct. Therefore, they (models) must be supplemented by conjugation conditions for unknown functions on such inclusions. In [15] a mathematical model of the motion of a fluid based on the Navier-Stokes equations in a region that is separated by a semipermeable membrane has been formulated. At the point of the existing membrane $(x=0)$ for the concentration of dissolved salts, the following conjugation conditions have been proposed:

$$
\begin{gathered}
-D_{c} \frac{d c^{-}}{d x}(0)+\sigma u c^{-}(0)=0, \\
-D_{c} \frac{d c^{+}}{d x}(0)+u c^{+}(0)=u(1-\sigma) c^{-}(0),
\end{gathered}
$$

where $\sigma \in[0 ; 1]$ coefficient that reflects the degree of membrane's ideality (the membrane is perfectly perfect if $\sigma=1$ ). Here $D_{c}$ is the diffusion coefficient, $u$ - the velocity of the fluid.

In articles [16] and [17] parabolic equations that describe the process of heat transfer in an area with a thin, weakly permeable inclusion with conjugation conditions such as "concentrated own source" have been considered. A new formulation of such problems in which the main parabolic equation changes to the system of differential equations of the first order with coefficients from the classes of generalized functions has been offered. The theorems of existence and uniqueness of the generalized solution have been proved.

The systemic problems of mathematical modeling of geomagnetic processes in heterogeneous media have been studied in [3-5]. In particular, the condition of con- 
jugation for the heads in the filtration problem is derived from the assumption that the heads $h$ linearly varies from $h^{-}$to $h^{+}$along the normal $n$ to the cross section of the thin inclusion $\gamma$ with the thickness $l$. That is

$$
h(\xi, t)=\frac{h^{+}-h^{-}}{l} \xi+h^{-}, \quad \frac{\partial h}{\partial \xi}=\frac{h^{+}-h^{-}}{l}, \quad 0 \leq \xi \leq l .
$$

Here $h^{+}$and $h^{-}$are the values of the pressure on inclusion under $\xi=l$ and $\xi=0$, correspondingly. The coordinate system $O \xi$ is tied to normal before inclusion. Then the boundary condition of conjugation of non-ideal contact has the form [3-5]

$$
\left.(\mathbf{u}, \mathbf{n})^{ \pm}\right|_{\gamma}=-k_{0} \cdot \frac{d h}{d \xi},
$$

or

$$
\left.(\mathbf{u}, \mathbf{n})^{ \pm}\right|_{\gamma}=-\frac{k_{0}}{l}[h] .
$$

In condition (1) $k_{0}$ is a value of filtration coefficient of a thin inclusion.

\section{Nonlinear Dependence of the Coefficient of Soil Moisture DifFusion on Physical AND ChEMICAL FACTORS AND PROBLEM STATEMENT}

One of the defining parameters included in the moisture transfer equation is the coefficient of soil moisture diffusion. It nonlinearly depends on the degree of saturation $s=\left(\theta-\theta_{\min }\right) /\left(\theta_{\max }-\theta_{\min }\right)$, concentration $c$ chemical substances in the porous liquid and temperature $T$. Here $\theta=V_{m} / V$ is the volumetric moisture; $V_{m}$ - the volume of porous liquid in the distinguished volume $V$ of soil; $\theta_{\min }$ - the residual (minimal) humidity (or quantity of liquid) which is left in soil after full drainage; $\theta_{\max }$ - the maximum humidity value. In the case of two component porous environment (gas component is abscent) $\theta_{\max }=n$, where $n$ is the soil porosity. Therefore, it is just $0 \leq \theta_{\min } \leq \theta \leq \theta_{\max }=n$. For determination of nonlinear functions $D(\theta, c, T)$ the following empirical dependencies can be used:

1. The model of R.H. Brooks and A.T. Corey [18]:

$$
D(\theta, c, T)=D_{0} s^{2+\frac{1}{\lambda}}, D_{0}=-\frac{K_{0}(c, T) \psi_{b}}{\lambda\left(\theta_{\max }-\theta_{\min }\right)},
$$

where $K_{0}(c, T)$ is the filtration coefficient, which depends nonlinearly on $c$ and $T$ [19]; $\lambda$ - the characteristic parameter of soil pores' size distribution; $\psi_{b}-$ the air pressure in soil. 
2. The model of Mualem-Van Genuchten [18]:

$$
D(\theta, c, T)=D_{0} \frac{\left[1-\left(1-s^{\frac{r}{\lambda}}\right)^{\frac{\lambda}{r}}\right]^{2}\left(1-s^{\frac{r}{\lambda}}\right)^{\frac{1}{r}-1}}{s^{\frac{1}{\lambda}+\frac{1}{2}}},
$$

where $r$ is an empirical parameter. There are also other dependencies for diffusion coefficient of soil moisture, some of them are presented in [20].

H. Gao and M. Shao made the conclusions [21] that saturated hydraulic conductivity $k_{s}$ significantly increased linearly with increasing temperature from $6^{\circ} \mathrm{C}$ to $36^{\circ} \mathrm{C}$. The $k_{s}$ value for lou soil at $36^{\circ} \mathrm{C}$ was 2.2 times grater than at $6^{\circ} \mathrm{C}$ and 2.35 times greater for sandy loess.

The permeability of mixtures of basalt soil and bentonite has been investigated in [22]. There were used salt solutions of $\mathrm{NaCl}$ and $\mathrm{CaCl}_{2}$ at five concentrations. For example the hydraulic conductivity of 100(the basalt soil): 20 (bentonite) mixture was increased from $2.5 \times 10^{-8} \mathrm{~cm} / \mathrm{s}$ to $2.3 \times 10^{-7} \mathrm{~cm} / \mathrm{s}$ while $\mathrm{NaCl}$ solutions concentration was increased from $0 \mathrm{~mol} / \mathrm{L}$ to $1 \mathrm{~mol} / \mathrm{L}$.

The three different inorganic salt solutions have been investigated in [23]: sodium chloride $(\mathrm{NaCl})$, calcium cloride $\left(\mathrm{CaCl}_{2}\right)$ and Magnesium Chloride $\left(\mathrm{MgCl}_{2}\right)$. There were used distilled water and salt solutions at four concentrations varying between 0.01 and $2 \mathrm{~N}$. The authors concluded that the hydraulic conductivity of soil samples increased by using salt solutions.

At present, studies of the influence of such dependencies of the moisture diffusion coefficient for the thin inclusion of these factors on the processes of moisture distribution. We put in perspective establishing the degree of such influence, as a target of research. To achieve this goal, we consider the problem in several stages. The first stage, which defines the purpose of this article, is the modification of conjugation conditions for semipermeable inclusions, taking into account such nonlinear dependencies.

It should be noted that in this article gas component of a porous medium is neglected. We suppose that changes of soil temperature are within the range of $0^{\circ} \mathrm{C}$ to $90^{\circ} \mathrm{C}$.

\section{Modified Conjugation Condition for Moisture}

First, we consider conjugation conditions for humidity $\theta$. We assume (due to the subtlety of inclusion) that the processes of moisture transfer in the cross section of this inclusion are stationary (or, at least, quasi-stationary). Thus, for inclusion let's consider the following problem of moisture transfer

$$
\frac{d}{d \xi}\left(-D_{\theta}(\theta, c, T) \frac{d \theta}{d \xi}+F_{\text {osm }}\right)=0, \quad 0<\xi<l,
$$




$$
\theta(0)=\theta^{-}, \quad \theta(l)=\theta^{+} .
$$

Here $c$ is the concentration of one component chemical porous liquid, $T$ - the temperature (determined functions are either known or they are obtained from solutions of relevant boundary problems of heat-salt transfer); $D_{\theta}(\theta, c, T)$ - the coefficient of soil moisture diffusion, which depends on moisture, salt concentration and temperature; $F_{\text {osm }}$ - the function of osmotic effects on the flow of moisture and can be defined as [19]

$$
F_{\text {osm }}=k_{c} \frac{d c}{d \xi}+k_{T} \frac{d T}{d \xi},
$$

where $k_{c}, k_{T}$ are the coefficients of chemical and thermal osmosis, respectively.

From the problem (2), (3) we have

$$
\begin{aligned}
& \theta(\xi)=\frac{\theta^{+}-\theta^{-}}{\int_{0}^{l} \frac{d x}{D_{\theta}(\theta, c, T)}} \int_{0}^{\xi} \frac{d x}{D_{\theta}(\theta, c, T)} \\
&-\frac{\int_{0}^{l} \frac{F_{\text {osm }}}{D_{\theta}(\theta, c, T)} d x}{\int_{0}^{l} \frac{d x}{D_{\theta}(\theta, c, T)}} \int_{0}^{\xi} \frac{d x}{D_{\theta}(\theta, c, T)} \\
&+\int_{0}^{\xi} \frac{F_{\text {osm }}}{D_{\theta}(\theta, c, T)} d x+\theta^{-} .
\end{aligned}
$$

It is easy to verify that function $\theta(\xi)$ satisfies equation (2) and conditions (3). Then we get

$$
\text { (4) } \begin{aligned}
\frac{d \theta}{d \xi}=\frac{\theta^{+}-\theta^{-}}{D_{\theta}(\theta, c, T) \int_{0}^{l} \frac{d x}{D_{\theta}(\theta, c, T)}} \\
-\frac{\int_{0}^{l} \frac{F_{\text {osm }}}{D_{\theta}(\theta, c, T)}}{D_{\theta}(\theta, c, T) \int_{0}^{l} \frac{d x}{D_{\theta}(\theta, c, T)}}+\frac{F_{\text {osm }}}{D_{\theta}(\theta, c, T)} .
\end{aligned}
$$

According to [4], we will formulate the conjugation condition on the basis of the law of preserving the flow of fluid through the cross-sectional area of the inclusion surface along the normal in the time unit. Since the flow

$$
\mathbf{u}=-D_{\theta}(\theta, c, T) \frac{d \theta}{d \xi}+F_{\text {osm }}
$$


and

$$
\mathbf{u}=\mathbf{u}^{+}=\mathbf{u}^{-}
$$

then

$$
\left.(\mathbf{u}, \mathbf{n})^{ \pm}\right|_{\gamma}=-D_{\theta}(\theta, c, T) \frac{d \theta}{d \xi}+F_{\text {osm }}
$$

From (4) we have

$$
-D_{\theta}(\theta, c, T) \frac{d \theta}{d \xi}+F_{\mathrm{osm}}=-\frac{[\theta]}{\int_{0}^{l} \frac{d x}{D_{\theta}(\theta, c, T)}}+\frac{\int_{0}^{l} \frac{F_{\mathrm{osm}}}{D_{\theta}(\theta, c, T)} d x}{\int_{0}^{l} \frac{d x}{D_{\theta}(\theta, c, T)}} .
$$

Here $[\theta]=\theta^{+}-\theta^{-}$denotes the leap of moisture on inclusion. From (6) and (5) we have the final formula of the modified condition of the conjugation of the non ideal contact for the moisture on the inclusion, the coefficient of soil moisture diffusion which depends on the physico-chemical factors and takes into account osmosis

$$
\left.(\mathbf{u}, \mathbf{n})^{ \pm}\right|_{\gamma}=-\frac{[\theta]}{\int_{0}^{l} \frac{d x}{D_{\theta}(\theta, c, T)}}+\frac{\int_{0}^{l} \frac{F_{\mathrm{osm}}}{D_{\theta}(\theta, c, T)} d x}{\int_{0}^{l} \frac{d x}{D_{\theta}(\theta, c, T)}} .
$$

If $D_{\theta}(\theta, c, T)=D_{0}=$ const, $F_{\text {osm }} \equiv 0$, then from condition (7) we get the classical conjugation condition of non-ideal contact [4].

\section{Modification of Conjugation Conditions For Salt Concentration AND TEMPERATURE}

For inclusion let's consider the following problem of heat transfer

$$
\begin{gathered}
-\frac{d}{d \xi}\left(\lambda(\theta) \frac{d T}{d \xi}\right)=0, \quad 0<\xi<l, \\
T(0)=T^{-}, \quad T(l)=T^{+} .
\end{gathered}
$$

Here $\lambda(\theta)$ is the coefficient of thermal conductivity of moist porous environment [24].

From the problem (8), (9) we have

$$
T(\xi)=\frac{T^{+}-T^{-}}{\int_{0}^{l} \frac{d x}{\lambda(\theta)}} \int_{0}^{\xi} \frac{d x}{\lambda(\theta)}+T^{-}
$$


and

$$
\frac{d T}{d \xi}=\frac{T^{+}-T^{-}}{\lambda(\theta) \int_{0}^{l} \frac{d x}{\lambda(\theta)}} .
$$

We will formulate the conjugation condition on the basis of the law of preserving the flow of heat through the cross-sectional area of the inclusion surface along the normal in a time unit. Since the flow

$$
\mathbf{q}_{T}=-\lambda(\theta) \frac{d T}{d \xi}+\rho c_{\rho} \mathbf{u} T
$$

then [4]

$$
\left.\left(\mathbf{q}_{T}, \mathbf{n}\right)^{ \pm}\right|_{\gamma}=-\frac{[T]}{\int_{0}^{l} \frac{d x}{\lambda(\theta)}}+\rho c_{p}(\mathbf{u}, \mathbf{n})^{ \pm} \frac{T^{+}+T^{-}}{2}
$$

Here $\rho, c_{p}$ stand for the density and the specific heat capacity of porous liquid; $[T]=$ $T^{+}-T^{-}$is the leap of temperature on inclusion.

Since inclusion has properties of a semipermeable membrane, then for the stream of dissolved substances we have

$$
(1-\sigma) \mathbf{q}_{c}=\mathbf{q}_{c}^{+}=\mathbf{q}_{c}^{-}
$$

Parameter $\sigma, 0 \leq \sigma \leq 1$, determines semipermeable properties of thin inclusion. If $\sigma=1$, then thin inclusion is an ideal semipermeable membrane. In the case of $\sigma=0$ inclusion $\gamma$ does not possess the properties of semipermeable membrane. It is known that (see Sections 2 and 3.1 in Ref. [10]): "The dimensionless quantity $\sigma$ will here be called membrane efficiency, but it also referred to as osmotic efficiency or reflection coefficient; it characterizes the ability to impede solute transport and depends on both the porous medium and the pore fluid as discussed below in Section 3.1." So we get

$$
\left.\left(\mathbf{q}_{c}, \mathbf{n}\right)^{ \pm}\right|_{\gamma}=(1-\sigma)\left(-\frac{[c]}{\int_{0}^{l} \frac{d x}{D_{c}(\theta, T)}}-\frac{\int_{0}^{l} \frac{D_{T}}{D_{c}(\theta, T)} \frac{d T}{d \xi} d x}{\int_{0}^{l} \frac{d x}{D_{c}(\theta, T)}}+\left(\mathbf{u}^{ \pm}, \mathbf{n}\right) \frac{c^{+}+c^{-}}{2}\right) .
$$

Here $D_{c}, D_{T}$ are the coefficients of diffusion and thermo-diffusion, respectively. 


\section{Summary AND Directions FOR Further RESEARCH}

The processes of non-isothermal moisture transfer of single-component chemical solutions in the porous environment containing thin semi-permeable clay inclusions have been considered. The conjugation conditions on the thin semi-permeable inclusion for humidity, salt concentration and temperature have been modified in the article. The modification is to take into account the dependence of the inclusion parameters on the physical and chemical factors, as well as the possible available osmosis. Research of the significance of the impact of such modifications requires numerical experiments for model problems. It requires the formulation of the corresponding boundary value problems, schemes of their numerical solution, program realization of algorithms and actually the calculations themselves. We have solved the corresponding moisture transport problem [25] but without taking into account salt and heat transfer. We are going to find numerical solution of a boundary value problem for moisture, salt solutions concentration and heat equations and to publish the results.

\section{REFERENCES}

[1] Ye.Ya. Chaplya, O.Yu. Chernukha, Yu.I. Bilushchak (2012) Contact Intial Boundary-Value Problem of the Diffusion of Admixture Particles in a TwoPhase Stochastically Inhomogeneous Stratified Strip. Journal of Mathematical Sciences 183(1) 83-89.

[2] O.R. Hachkevych, R.M. Kushnir (2018) Selected Problems of the Mechanics of Coupled Fields. Journal of Mathematical Sciences 229(2) 115-132.

[3] V.S. Deineka, I.V. Sergienko (2001) "Models and Methods of Solving Problems in Inhomogeneous Media". Kiev (in Russian).

[4] I.V. Sergienko, V.V. SKOPetSk yi, V.S. DeineKa (1991) "Mathematical Modeling and Research of Processes in Heterogeneous Environments". Kiev (in Russian).

[5] I.V. Sergienko, V.S. Deineka (2005) "Optimal Control of Distributed Systems with Conjugation Conditions". Springer-Verlag New York Inc.

[6] K.M. SAmPle-Lord, C.D. ShaCKelford (2014) Membrane Behavior for Unsaturated Bentonite Barriers. In: A.J. Puppala, P. Bandini, T.C. Sheahan, M. Abu-Farsakh, Xiong Yu, L.R. Hoyos (Eds) “Geo-Congress 2014 Technical Papers”. American Society of Civil Engineers; Cdr edition, pp. 1900-1909.

[7] S.-S. Yeo, C.D. Shackelford, J.C. Evans (2005) Membrane Behavior of Model Soil-Bentonite Backfills. Journal of Geotechnical and Geoenvironmental Engineering 131 416-429.

[8] J.C. Evans, C.D. Shackelford, S.-S. Yeo, J. Henning (2008) Membrane Behavior of Soil-Bentonite Slurry-Trench Cutoff Walls. Soil \& Sediment Contamination 17 316-322. 
[9] F.M. AzAD, D.W. Airey, R.K. Rowe, A. El-ZEIn (2010) Laboratory Investigation of Moisture Content Redistribution on the Base of Double Composite Liner Systems. In: Proceedings of the 6th International Congress on Environmental Geotechnics, New Delhi, India, pp. 354-359.

[10] C.E. NeuzIL, M. Person (2017) Reexamining Ultrafiltration and Solute Transport in Groundwater. Water Resources Research 53 4922-4941.

[11] A. REVIL (2017) Transport of Water and Ions in Partially Water-Saturated Porous Media. Part 2. Filtration Effects. Advances in Water Resources 103 139-152.

[12] G.L. Bohnhoff, C.D. Shackelford (20150 Salt Diffusion Through a BentonitePolymer Composite. Clays and Clay Minerals 63 145-162.

[13] T.M. Whitworth, A. Ghazifard (2009) Membrane Effects in Clay-Lined Inward Gradient Landfills. Applied Clay Science 43 248-252.

[14] S.L. Barbour, D.G. Fredlund (1989) Mechanisms of Osmotic Flow and Volume Change in Clay Soils. Can. Geotech. J. 26 551-562.

[15] J. Hron, M. Neuss-Radu, P. Pustejovsk (2010) Mathematical Modeling and Simulation of Flow in Domains Separated by Leaky Semipermeable Membrane Including Osmotic Effect. Preprint no. 2010-35, Mathematical Institute of the Charles University, Prague, Czech Republic, 16 p.

[16] D.A. NomirovskiI, O.I. Vostrikov (2016) Generalized Statements and Properties of Models of Transport Processes in Domains with Cuts. Cybernetics and Systems Analysis 52 931-942.

[17] O.I. Vostrikov, D.A. NomirovskiI (2016) Generalized Solvability of Transmission Systems in a Layered Domain with the Condition of a "Proper Lumped Source". Journal of Computational \& Applied Mathematics 1 19-27.

[18] J.-G. Caputo, Y.A. Stepanyants (2007) Front Solutions of Richards' Equation. Transport in Porous Media 74 1-20.

[19] P.M. Martyniuk, T.V. Kutia, O.P. Ostapchuk, O.L. Pinchuk (2018) Filtration Equation and Movement of the Wetting Interface in Case of Pressure Pipeline Breakthrough Under the Conditions of Variable Porosity. JP Journal of Heat and Mass Transfer 15 281-293.

[20] F.J. LeiJ, W.B. Russell, S.M. Lesch (1997) Closed Form Expressions for Water Retention and Conductivity Data. Ground Water 35 848-858.

[21] H. GaO, M. Shao (2015) Effects of Temperature Changes on Soil Hydraulic Properties. Soil \& Tillage Research 153 145-154.

[22] A.K. Mishra, M. Ohtsubo, L. Loretta, T. Higashi (2005) Effect of Salt Concentrations on the Permeability and Compressibility of Soil-Bentonite Mixtures. Journal of the Faculty of Agriculture, Kyushu University 50(2) 837-849.

[23] N. Shariatmadari, M. Salamani, M.K. Fard (2011) Effects of Inorganic Salt Solutions on Some Geotechnical Properties of Soil-Bentonite Mixtures as Barriers. International Journal of Civil Engineering 9(2) 103-110. 
[24] M. Sadeghi, B. Ghanbarian, R. Horton (2018) Derivation of an Explicit Form of the Percolation-Based Effective-Medium Approximation for Thermal Conductivity of Partially Saturated Soils. Water Resources Research 54(2) 1389-1399.

[25] P.M. Martyniuk, O.R. Michuta, O.V. Ulianchuk-Martyniuk, M.T. Kuzlo (2018) Numerical Investigation of Pressure Head Jump Values on a Thin Inclusion in One-Dimensional Non-Linear Soil Mousture Transport Problem. International Journal of Applied Mathematics 31(4) 649-660. 